\title{
Impurity-Related Nonlinear Optical Absorption in Delta-Doped Quantum Rings
}

\author{
R.L. Restrepo ${ }^{a}$, J.C. Martínez-Orozco ${ }^{b}$, M.G. BARseghyan $^{c}$, M.E. Mora-Ramos $^{a, d, e}$ \\ AND C.A. DUQUE ${ }^{e}$ \\ ${ }^{a}$ Física Teórica y Aplicada, Escuela de Ingeniería de Antioquia, AA 7516, Medellín, Colombia \\ ${ }^{b}$ Unidad Académica de Física, Universidad Autónoma de Zacatecas, CP 98060, México \\ ${ }^{c}$ Department of Solid State Physics, Yerevan State University, 0025 Yerevan, Armenia \\ ${ }^{d}$ Facultad de Ciencias, Universidad Autónoma del Estado de Morelos, CP 62209, México \\ ${ }^{e}$ Grupo de Materia Condensada-UdeA, Instituto de Física, Facultad de Ciencias Exactas y Naturales \\ Universidad de Antioquia UdeA, Calle 70 No. 52-21, Medellín, Colombia
}

\begin{abstract}
Using a variational procedure within the effective mass approximation, we have calculated the hydrogenic impurity binding energy as well as the impurity-related nonlinear optical absorption in a single quantum ring with axial $n$-type delta-doping. The delta-like potential along the $z$-direction is analytically modeled with a Hartree-like confining profile fitted to a one-dimensional Thomas-Fermi-based potential in the local density approximation. Both on-center and on-edge impurity positions are considered and the energies of the impurity states are examined as functions of the vertical size of the ring. It is found that the effect of changes in the geometry of the quantum ring leads to a shifting of the resonant peaks of the intra-band optical spectrum.
\end{abstract}

DOI: $10.12693 /$ APhysPolA.125.245

PACS: 42.65.-k, 73.21.-b, 78.67.-n

\section{Introduction}

The study of the electronic properties of hydrogen-like impurities in low-dimensional semiconductor heterostructures attracts much interest given the vast possibility of purposeful manipulation of the impurity binding energy by means of external influences with the possibility of controlling the electronic and optical properties of functional devices based on such heterostructures [1]. The simultaneous effect of impurity and external influences on the linear and nonlinear optical properties of semiconductor nanostructures has also been considered in recent years $[2,3]$. So far, there are only a few published research articles related with the effects of impurity and external influences on linear and nonlinear optical properties of semiconductor quantum rings (QRs) [4, 5]. On the other hand, the use of delta-doping techniques allows the creation of high-density two-dimensional electron gases [6-8], providing a suitable environment for the increase in the conductivity. But the enlargement of the carrier concentration can also lead to the amplification of nonlinear optical responses.

In consequence, our purpose here is to investigate the optical absorption in delta-doped QRs. In Sect. 2 we give a brief outline of the theoretical model used. This is followed by Sect. 3 containing the presentation and discussion of the results obtained. Finally, the conclusions of the work are briefly stated in Sect. 4.

\section{Outlook on the theoretical model}

The system under study is a GaAs-based cylindrical QR with inner and outer radii $R_{1}$ and $R_{2}$, respectively, and vertical size $L<R_{2}$. The potential energy configuration considered includes infinite barriers at $r<R_{1}$ and $r>R_{2}$, and for $|z|>L / 2$. The potential function is zero elsewhere with the exception of the region $|z|<L / 2$. In this interval the model assumes the presence of an $n$-type delta-doped layer placed at $z=0$, which causes a conduction band bending. The situation under study, although similar to the usual one of a single layer — or narrow slab - of donor dopant atoms, differs from it in the fact that the radial boundary conditions are now restricted by the finite dimension of the heterostructure. Therefore, the correct description of the potential function must include a self-consistent solution involving a three-dimensional Poisson equation. We avoid this complication by proposing an approximate model in which the potential profile associated with the delta-doped quantum well is analytically described using a Lorentzian function with two parameters. These parameters are then fitted to reproduce the depth of the quantum well obtained within a self-consistent one-dimensional local-density theory in the Thomas-Fermi approximation. The density of ionized impurities is taken as $N_{2 \mathrm{~d}}=7.5 \times 10^{12} \mathrm{~cm}^{-2}$.

We shall consider two values of the hydrogenic impurity axial position: one is the central value $z=0$ (hereafter named on-center). The second is the edge of the QR, $z=L / 2$ (hereafter named on-edge). After solving the Schrödinger-like problems for the motion along the $z$-axis and the in-plane orientation $[9,10]$, the resulting states are included in a variational calculation with hydrogenic type trial wave function to obtain the impurity states and, with these states, the linear and nonlinear optical absorption coefficients are evaluated as functions of the incident photon energy [11].

\section{Results and discussion}

Figure 1 contains the calculated binding energy of the $1 s$ and $2 p_{x}, 2 p_{z}$ impurity states as functions of the vertical QR size, $L$. In the calculation, the values $R_{1}=2 \mathrm{~nm}$ 
and $R_{2}=10 \mathrm{~nm}$ have been used. In addition, the impurity atom is assumed to be placed at $z_{i}=0$. Negative values of the binding energies associated to $2 p$ states are due to the particular energy reference value, chosen to describe the $1 s$-like situation. The slight decreasing variation of the on-center $1 s$ - and $2 p_{x}$-like impurity binding energies is explained by the small sensitivity of these orbitals with respect to the $z$-component of the uncorrelated electron ground state wave function. This sensitivity augments when the impurity atom locates at the QR edge; thus leading to the more pronounced fall of $E_{b}$ observed in Fig. 1d,e.

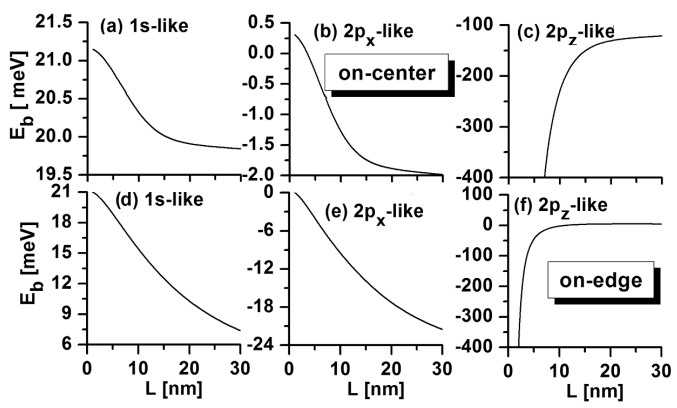

Fig. 1. Impurity binding energy in a GaAs quantum ring with a delta-doped layer at $z=0$ as a function of the vertical size of the ring. Impurity states $1 s, 2 p_{x}$, and $2 p_{z}$ are considered for both on-center, and on-edge impurity positions.

The strong confinement of the electron ground state in the vertical direction contributes to the strengthening of the Coulomb interaction. For that reason, the magnitude of the binding energy associated with the $2 p_{z}$ orbitals is significantly large for small $L$ values. Augmenting the vertical size of the $\mathrm{QR}$ is reflected in the strong fall in the strength of the Coulombic coupling, provided that the $z$-dependent part of the uncorrelated electron wave function loses confinement. This is more apparent in the on-edge case.

Figures 2 and 3 show the calculated linear, nonlinear, and total optical absorption coefficients related with the $1 s-2 p_{x}$ energy transition for $x$-polarization of the incident radiation. It is possible to detect a blueshift in the resonant peak position associated with the displacement of the vertical impurity position from the $z=0$ plane upwards (Fig. 2). This shift is accompanied by an increase of the linear and nonlinear peak amplitudes coming from the increment in the resonant frequency and the growth of the expected value of the electric dipole moment $M_{1 s, 2 p_{x}}=\left\langle\psi_{1 s}|e x| \psi_{2 p_{x}}\right\rangle$.

In a configuration with fixed $L, R_{2}$, and $z_{i}$, the variation of the size of the inner radius produces the results depicted in Fig. 3, for the linear, nonlinear, and total optical absorption coefficient as a function of the incident photon energy. Contrary to the previous case, the increase in the inner radius has the consequence of a redshift in the positions of the resonant peaks. This is the result of the reduction in the impurity interstate energy differences (although the energy of each individual state

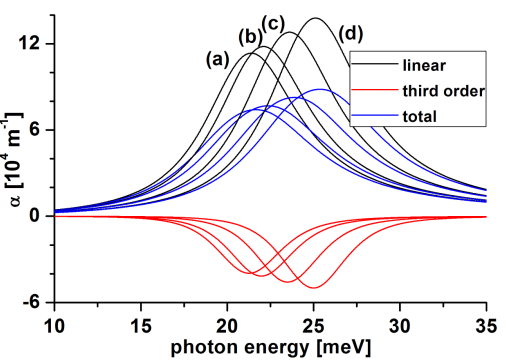

Fig. 2. Optical absorption coefficient $\left(1 s-2 p_{x}\right.$ transitions with $x$-polarization of the incident radiation) in GaAs delta-doped quantum ring as a function of the incident photon energy. The quantum ring configuration has $R_{1}=2 \mathrm{~nm}, R_{2}=10 \mathrm{~nm}, L=5 \mathrm{~nm}$; with four different values of the vertical impurity position $z_{i}$ : 0 (a), $1.5 \mathrm{~nm}(\mathrm{~b}), 3 \mathrm{~nm}$ (c), and $4.5 \mathrm{~nm}(\mathrm{~d})$.

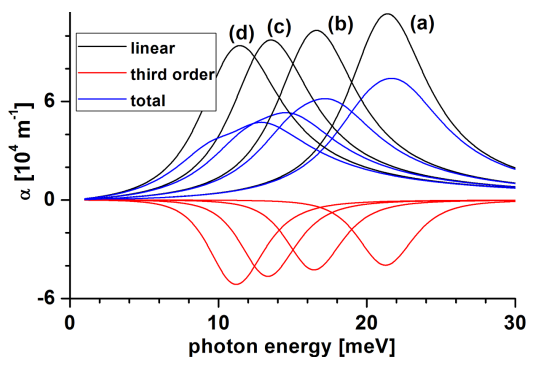

Fig. 3. Optical absorption coefficient for $1 s^{-} 2 p_{x}$ transitions with $x$-polarization of the incident radiation in GaAs delta-doped quantum ring as a function of the incident photon energy. The quantum ring configuration has $R_{2}=10 \mathrm{~nm}, L=5 \mathrm{~nm}$, and $z_{i}=0$; with four different values of the inner radius $R_{1}: 2 \mathrm{~nm}(\mathrm{a}), 3 \mathrm{~nm}(\mathrm{~b})$, $4 \mathrm{~nm}(\mathrm{c})$, and $5 \mathrm{~nm}(\mathrm{~d})$.

grows as a function of the inner radius, the $1 s$-like increases with a higher rate). However the effect over the amplitudes is different in the linear and nonlinear terms. In the linear case the resonant peak amplitude decreases whereas the - negative - magnitude of the third-order nonlinear resonant peak augments as long as there is an increment of the inner radius. This can be explained by the combination of two factors: a reduction in the absolute value of the interstate matrix element, and the increase in the absolute difference $\left|M_{1 s, 1 s}-M_{2 p_{x}, 2 p_{x}}\right|$.

Figures 4 and 5 show the effect of the application of hydrostatic pressure, $P$, on the linear, nonlinear, and total contributions to the optical absorption coefficient. A fixed geometrical configuration has been selected and we have included four different values of $P$ in the calculation. Figure 4 corresponds to the optical absorption associated with the $1 s-2 p_{x}$ energy transition with $x$-polarization of the incident radiation, whereas Fig. 5 contains the results for the absorption that involves the $1 s-2 p_{z}$ impurity energy transition with $z$-polarization of the incident radiation.

The influence of the hydrostatic pressure is introduced via the corresponding dependences of the electron effective mass and the dielectric constant on $P: m^{*} / m_{0}=$ 


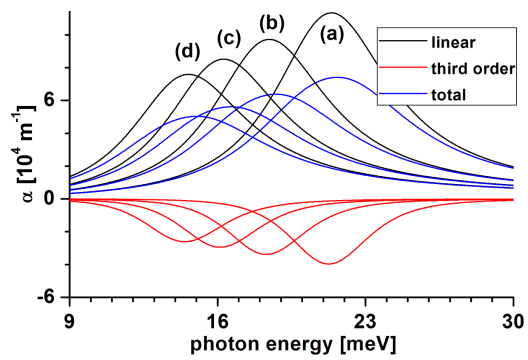

Fig. 4. Optical absorption coefficient $\left(1 s-2 p_{x}\right.$ transitions with $x$-polarization of the incident radiation) in GaAs delta-doped quantum ring as a function of the incident photon energy. The quantum ring has a zero-pressure geometrical configuration with $R_{1}=2 \mathrm{~nm}$, $R_{2}=10 \mathrm{~nm}, L=5 \mathrm{~nm}$, and $z_{i}=0$; with four different values of the applied hydrostatic pressure: 0 (a), 20 kbar (b), 40 kbar (c), and 60 kbar (d).

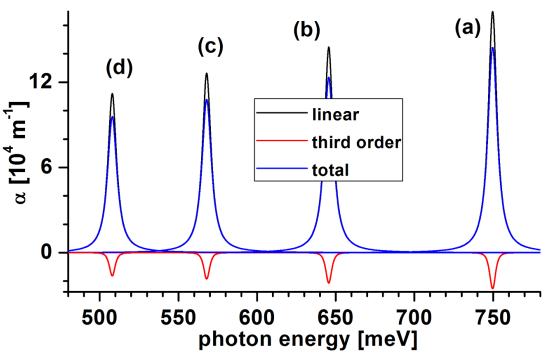

Fig. 5. As Fig. 4, for $1 s-2 p_{z}$ transitions and $z$-polarization.

$0.0665+5.7076 \times 10^{-4} P, \varepsilon=12.25-0.027 P$. Radial and vertical dimensions change according the following expressions: $R(P)=R(0)\left[1-2\left(S_{11}+2 S_{12}\right) P\right]^{1 / 2}$, $L(P)=L(0)\left[1-\left(S_{11}+2 S_{12}\right) P\right][12]$.

At a first glance, the effect of the hydrostatic pressure is to induce a redshift in the optical absorption resonant peak position. The shifting is more noticeable in the case of the $1 s-2 p_{z}$ transition. It is related, in both cases, to a competition between the reduction in the spatial extension of the system, the overall decrease of the effective Bohr radius, and the effective growth of the strength of the Coulombic interaction. This results in lower values of the inter-state energy difference. Here, one must take into account that, although the uncorrelated electron energies in the vertical motion are increasing functions of $P$, the distance between the states becomes smaller as long as the pressure increases. This effect is much less pronounced in the case of the radial motion. On the other hand, the fall in the resonant peak amplitudes associates mostly with the increase in both radial and vertical electron state confinement. This makes the contribution of lower values of radial and $z$-coordinates to dominate in the integrand of the transition electric dipole moment matrix elements.

\section{Conclusions}

In this work we have studied some features of the linear and nonlinear optical absorption associated to donor impurity states in a GaAs cylindrical quantum ring with an inserted in-plane delta-doped layer. The binding energies of $1 s$ and $2 p$ states are reported as functions of the ring's vertical size. The optical absorption resonant peaks show a blueshift when the position of the hydrogenic impurity atom along the vertical direction is shifted upwards. On the other hand, when geometry of the system changes by augmenting the inner radius, keeping fixed the remaining dimensions, the resonant peaks positions exhibit a redshift.

The application of hydrostatic pressure has the effects of inducing a redshift of the optical absorption resonant peak positions as well as the decrease of the corresponding peak amplitudes. This relates with the strengthening of the Coulomb electron-impurity interaction, the reduction in the uncorrelated energy difference and the growth in electron wave function confinement.

\section{Acknowledgments}

M.E.M.R. acknowledges support from Mexican CONACYT through grant CB-2008-101777, and through sabbatical grant 2011-2012 No. 18036. $\mathrm{He}$ also thanks Universidad de Antioquia and Escuela de Ingeniería de Antioquia for hospitality during his sabbatical stay. This research was partially supported by Colombian Agencies: CODI-Universidad de Antioquia (Estrategia de Sostenibilidad 2013-2014 de la Universidad de Antioquia and the project: E01535-Efectos de la presión hidrostática y de los campos eléctrico y magnético sobre las propiedades ópticas no lineales de puntos, hilos y anillos cuánticos de GaAs-(Ga,Al)As y $\left.\mathrm{Si} / \mathrm{SiO}_{2}\right)$, Facultad de Ciencias Exactas y NaturalesUniversidad de Antioquia (CAD-exclusive dedication project 2012-2013), and by El Patrimonio Autónomo Fondo Nacional de Financiamiento para la Ciencia, la Tecnología y la Innovacion, Francisco José de Caldas. The work was developed with the help of CENAPAD-SP, Brazil.

\section{References}

[1] A.D. Yoffe, Adv. Phys. 50, 1 (2001).

[2] W. Xie, Phys. Lett. A 372, 5498 (2008).

[3] I. Karabulut, S. Baskoutas, J. Appl. Phys. 103, 073512 (2008).

[4] W. Xie, Phys. Status Solidi B 246, 1313 (2009).

[5] W. Xie, Phys. Scr. 85, 055702 (2012).

[6] G.H. Döhler, Surf. Sci. 73, 97 (1978).

[7] L. Ioratti, Phys. Rev. B 41, 8340 (1990).

[8] L.M. Gaggero-Sager, J. Math. Chem. 25, 317 (1999).

[9] M.E. Mora-Ramos, C.A. Duque, Physica B 415, 72 (2013).

[10] J.M. Llorens, C. Trallero-Giner, A. García-Cristobal, A. Cantarero, Phys. Rev. B 64, 035309 (2001).

[11] D. Ahn, S.-L. Chuang, IEEE J. Quantum Electron. QE23, 2196 (1987).

[12] N. Raigoza, A.L. Morales, A. Montes, N. Porras-Montenegro, C.A. Duque, Phys. Rev. B 69, 045323 (2004). 